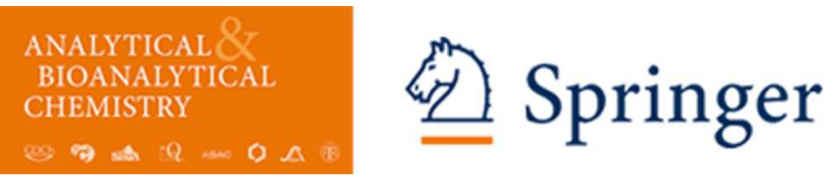

\title{
Post-translational modifications in tumor biomarkers: the next challenge for aptamers?
}

\begin{tabular}{|r|l|}
\hline Journal: & Analytical and Bioanalytical Chemistry \\
\hline Manuscript ID & ABC-01979-2017.R1 \\
\hline Type of Paper: & Feature Article \\
\hline Date Submitted by the Author: & n/a \\
\hline Complete List of Authors: & $\begin{array}{l}\text { Díaz-Fernández, Ana; Universidad de Oviedo Facultad de Quimica, Química } \\
\text { Física y Analítica } \\
\text { Miranda Castro, Rebeca ; Universidad de Oviedo Facultad de Quimica, } \\
\text { Química Física y Analítica } \\
\text { de los Santos Álvarez, Noemi; Universidad de Oviedo, Dpto Química Física } \\
\text { y Analítica } \\
\text { Lobo-Castañón, Maria Jesus; Universidad de Oviedo, Quimica Fisica y } \\
\text { Analitica }\end{array}$ \\
\hline Keywords: & \begin{tabular}{l} 
Aptamers, Cancer diagnostics, Glycans, Tumor biomarkers \\
\hline
\end{tabular} \\
\hline
\end{tabular}




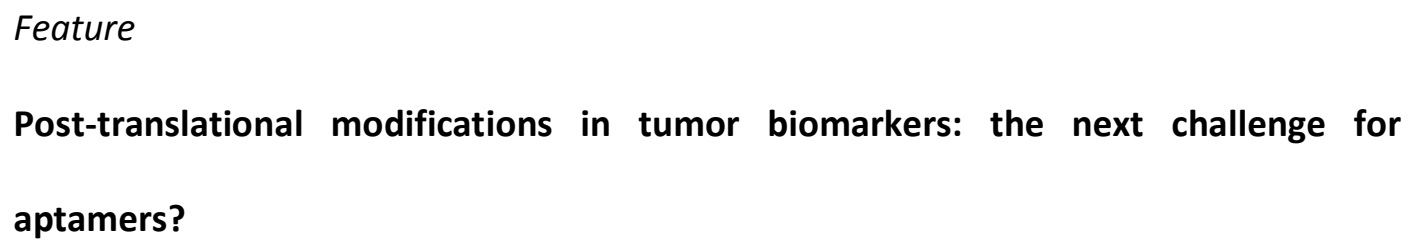

Dpto. Química Física y Analítica, Universidad de Oviedo, Julián Clavería 8, 33006

Oviedo, Spain.

* Corresponding Author. Email address: mjlc@uniovi.es 


\begin{abstract}
Advances in proteomics have fueled the search for novel cancer biomarkers with higher selectivity. Differential expression of low abundant proteins has been the usual way of finding those biomarkers. The existence of a selective receptor for each biomarker is compulsory for their use in diagnostic/prognostic assays. Antibodies are the receptors of choice in most cases although aptamers are becoming familiar because of their facile and reproducible synthesis, chemical stability as well as comparable affinity and selectivity. In recent years, it has been reported that the pattern of post-translational modifications, altered under neoplastic disease, is a better predictive biomarker than the total protein level. Among others, abnormal glycosylation is attracting great attention. Lectins and antibodies are being used for identification and detection of the carbohydrate moiety with low level of discrimination among various glycoproteins. Such level of selectivity is critical to bring next-generation biomarkers to the clinic. Aptamers that can be rationally tailored for a certain molecule domain can become the golden receptor to specifically detect aberrant glycosylation at each protein or even at each glycosylation site, providing new diagnostic tools for early detection of cancer.
\end{abstract}

Keywords: aptamers, cancer diagnostics, glycans, tumor biomarkers 


\section{Glycosylation: a distinctive feature of cancer}

The addition of carbohydrate moieties (glycans) to proteins in the endoplasmatic reticulum ( $\mathrm{N}$-linked glycans) or in the Golgi (O-linked glycans) is one of the most frequent posttranslational events along with phosphorylation [1]. In fact, between 70 and $80 \%$ of human proteins are glycosylated [2]. Protein synthesis is led by the genomic code but the functional properties are modulated by the glycan structure. A single protein can naturally carry different glycan chains, so they exist in several glycoforms, which confers a diversity of properties with important physiological implications. The presence of side sugar chains is particularly common in proteins located in the cell surface and in the extracellular matrix and has a decisive role in the cell communication including cell adhesion, signal trafficking, extravasation, metastasis or escaping from the immune system as well as in their stability (protection from proteases) [1]. The extension of protein glycosylation is dependent on the number of glycosylation sites in the protein sequence and the expression/activity of the enzymes involved, mainly glycosyltranferases and glycosidases, in the cell. The diverse and complex glycan structures, the glycome, make challenging their identification and quantitation, which is becoming essential to elucidate the significance in pathological conditions.

The natural variety of glycans is extended during malignant transformation of cells when the normal glycosylation machinery is altered ${ }_{L}$-for example up or down regulation of the enzymes involved, leading to changes in the chemical structure of the glycan (Figure 1). Main modifications are related to changes in linkage, hiperbranching of $\mathrm{N}$-glycans or truncation of Oglycans generating sialylated tumor antigens but not restricted to them. The level of glycosylation is also of paramount importance. The degree of occupancy at each site or the extension of the presence of defined structures such sialyl derivatives or core fucoses can vary. In fact, sialylation and fucosylation are the major glycosylation changes and their level is closely related with cancer biology through modulation of several biological processes $[3,4]$. Though aberrant glycosylation is not included in the eight hallmarks of cancer there is a large 
body of evidence showing that glycans are involved in the acquisition of all these distinct tumors capabilities [5]. Some of the glycosylation changes are only specific of tumors so these unique alterations in the glycosylation pattern may serve as a distinct feature of cancer cell, providing novel diagnostic tools. Therefore, the research attention is starting to move to clinically applicable glycan features to diagnose, predict recurrence/survival (prognostic) or predict the response to therapy to aid in the implementation of a personalized medicine.

To date, the majority of FDA-approved cancer biomarkers are glycoproteins or tumorassociated carbohydrate antigens (TACAs). In general, they lack sufficient specificity (absence of false positives). Even the most successful serum biomarker, the prostatic specific antigen (PSA) that was a milestone in the diagnostic of prostatic cancer, is not specific because it is elevated in benign diseases with a grey zone of diagnostic for values between the cut-off ( 4 $\mathrm{ng} / \mathrm{mL}$ ) and $10 \mathrm{ng} / \mathrm{mL}$. To improve sensitivity and specificity, the study of glycoforms of current biomarkers or of new ones is a promising approach. A successful case is the fucosylated $\alpha$ fetoprotein whose concentration in serum is more informative to discriminate hepatocellular cancer from other benign liver diseases than total $\alpha$-fetoprotein itself [3]. Evidences of correlation between altered glycosylation and tumor initiation, progression, and metastasis pose a persistent analytical challenge, the development of specific analytical assays capable of recognizing the altered glycosylated target molecule as new means for early cancer diagnosis. Receptor-based assays, with emphasis in aptamer-assays that have especial potential to meet this challenge, are the focus of this feature.

\section{Receptors for glycan structures}

The discovery of novel serum biomarkers is of paramount importance to deliver personalized cancer therapies. However, once a potential biomarker is identified, the corresponding analytical method has to be developed for its use in routine clinical practice. While glicoprofiling studies rely on enzymatic restriction, chromatographic separation and mass spectrometry analysis [6], the point-of-care detection of a defined biomarker requires 
other approaches. Ideally, detection methods should fulfill the ASSURED criteria (affordable, sensitive, specific, user-friendly, rapid and robust, equipment free and delivered to those who need it). To comply with it, receptor-based assays are the methods of choice for current biomarkers, mainly immunoassays. Usually, tumor biomarkers are in minute amounts surrounded by other proteins in concentrations several orders of magnitude higher. Therefore, high affinity biorecognition elements are needed to achieve the high sensitivity demanded. But the challenge to recognize a small portion of the glycan that is under/overexpressed in malignant cells is even more demanding, and the recognition of changes in glycosylation patterns requires novel and more specific receptors, specifically directed towards the glycan epitopes.

Boronic acid derivatives recognize diols in the glycan structure (Figure 2) and have been used since mid-20th century but they have their own intrinsic preferences unless additional functionalities are added to confer extra selectivity [7].

Nature also provides carbohydrate-reactive testing reagents, schematically represented in Figure 2 . It is possible to obtain highly specific anti-carbohydrates monoclonal antibodies [8], although many interesting glycan peptides are conserved among species and, therefore may be non-immunogenic for the mammals that often serve as vehicles for the production of monoclonal antibodies. In consequence, other vertebrates more evolutionarily distant from humans have been explored to obtain carbohydrate-binding antibodies [9]. Furthermore, antibody-carbohydrate interaction is weaker than antibody-protein one limiting the sensitivity of the assays.

Lectins, a large family of proteins of non-immune origin, are by far the most widely used receptor for glycan identification. They are isolated from natural sources in a great variety (hundreds) and show high selectivity toward defined glycosidic linkages between saccharides, thus providing structural information. They are the natural binders of carbohydrates in-vivo due to their multivalency though the overall affinity is modest $\left(10^{-4}-10^{-7}\right.$ 
M) in comparison with antibodies. The source and purity of lectins could be an issue to obtain high quality data. These features along with moderate cross-reactivity make the development of glycan-peptide antibodies preferable for analytical developments.

It is well recognized that neoplastic transformations nearly always imply alterations in glycan epitopes. However, many proteins secreted from tumor cells share similar changes in glycan expression. As an example above 650 core fucosylated (CF) sites were found in less than $330 \mathrm{CF}$ proteins in human sera or tumor cell lines but a small proportion (less than 50 peptides) showed altered level of fucosylation to discriminate pancreatic cancer [10, 11]. This means that receptors must recognize the specific aberrant glycan at each specific site, that is, information from both carbohydrate and peptide chains is needed. Currently, those types of receptors are not available and directing antibodies to a certain region comprising both protein and glycan moieties is not an easy task. Meanwhile, mixed antibody (for protein recognition) and lectin (for glycan alteration recognition) ELISA-like assays have been developed [12].

\section{Aptamers for cancer glycosylated biomarkers}

The ideal receptor for aberrant glycosylation such as core fucosylation should be capable of tightly bind both the amino acids near the glycosylation site and the glycan structure. Aptamers, single-stranded oligonucleotides obtained by an in vitro selection process to bind a specific target, have unique advantages to fulfil these requirements. Their small size, $\sim 13 \mathrm{kDa}$ for a 40 nucleotides aptamer, may favor the access to glycan epitopes. They are selected in a versatile and purely (bio)chemical way, which can be designed to enhance the affinity to the subnM range as well as to drive it toward a defined region of interest through convenient and smartly designed counter selections steps. Once selected, they are obtained on larger scales than antibodies, by a chemical process that reduces the batch-to-batch variability that may lead to dramatically different results. In consequence, a variety of aptamers targeting different kind of carbohydrates such as monosaccharides, oligo- and poly-saccharides, aminoglycoside 
antibiotics and glycoproteins have been described during the past quarter century $[13,14]$. Despite sugars are not considered to be highly aptagenic because theirs structure has few charged groups and aromatic ring moieties, discrimination of a single functional group has been described for carbohydrate compounds. The aptamer for the aminoglycoside antibiotic neomycin B binds 100 times more weakly the analogue paramomycin (substitution of $\mathrm{NH}_{2}$ with $\mathrm{OH}$ ) and its discrimination power remains when it is O-methylated to increase nuclease resistance in biological fluids [15].

Right after discovery, the non immunogenic nature of aptamers boosts the interest as therapeutics molecules but also as a novel analytical reagent for diagnostics. Clinical targets were rapidly screened and aptamers for them are currently available [14]. Aptamers for potential tumor biomarkers including some of those approved for clinical usage have been also described. Two different approaches have been devised for the selection of aptamers recognizing these targets: i) using an individual purified form of the target or cell overexpressing the target; ii) raising aptamers against unknown abnormal targets on the surface of tumor cells. The latter strategy called Cell-SELEX is now preferred though it requires an extra effort if knowledge of the target is finally needed. For cancer diagnostics serum proteins are the preferential targets to keep in mind non-invasive monitoring, so the first strategy is the only option.

Until now, most aptamers against glycoprotein biomarkers have been obtained without directing the selection towards any specific region of the target. Consequently, in most cases it is not known whether the glycan, the peptide or both are involved in the binding. The difficulty in fully characterize the aptamer-ligand complex is delaying this piece of relevant information. Thrombin aptamers are one of the few well characterized ones, though it is not a biomarker, and the glycosylated site is far from the aptamer recognition site [16]. Aptamers evolved against short synthetic peptides or recombinant proteins expressed in non-human cells cannot recognize the sugar chain because bacterial can not incorporate them. Only those 
evolved using purified human proteins or recombinant peptides expressed in human cell lines might present such ability. However, until now, only a few aptamers were tested for sugar discrimination. An aptamer for epidermal growth factor receptor variant III EGFRvIII (uniquely expressed in glioblastoma) purified from E. coli did not show any binding affinity for its highly glycosylated human counterpart but resulted of therapeutic interest because can be internalized into the cell inducing apoptosis. The transfected aptamer blocks the unglycosylated form of EGFRvIl recently synthesized inside the cell, which cannot undergo proper glycosylation reducing the amount of the cell membrane glycosylated protein, demonstrating that aptamers can disrupt post-translational modifications of proteins [17].

Aptamers have also shown the ability to distinguish between forms of the same protein with slightly different post-translational modifications. This is the case of the aptamer recognizing cyclophilin $\mathrm{B}(\mathrm{CypB})$, selected using as a target the secretome of pancreatic cancer instead of an individual target. The aptaassay developed using this aptamer as recognition element discriminated sera from pancreatic cancer patients and healthy volunteers but failed to correlate with the СурB levels detected by an immunoassay. This fact is attributed to the recognition by the aptamer of the human form of the protein but not the one expressed in bacteria while antibody interacts with both [18].

Ultimately, the success of obtaining very strong binders that differentiate subtle differences in glycans would depend on the availability of adequate glycopeptides. Initially, it was thought that conventional SELEX protocols cannot achieve such level of directed selection and carbohydrates alone were the target. In 2001 a RNA aptamer towards sialyl Lewis X antigen overexpressed in many tumors was evolved with a surprisingly low dissociation constant $\left(K_{d}=3.3 \mathrm{nM}\right)$ but the original DNA pool already had a relatively high affinity $\left(K_{d}=170\right.$ nM) [19]. Selectivity against other Lewis antigens was only 5 to 10 times. After this early success, aptamers derived against mono-, di- and oligosaccharides showed high dissociation constants because they lack charged groups or aromatic rings that are the source of 
hydrophobic, ionic and hydrogen-bonding main interactions of aptamers with their ligands. Besides, the intrinsic flexibility of carbohydrate is less amenable to strong binding. More recently, unmodified RNA and DNA aptamers for negatively-charged sialic acid $\mathrm{N}$ acetylneuraminic acid (Neu5Ac) and a tumor-related derivative $\mathrm{N}$-glycolylneuraminic acid (Neu5Gc) exhibited 1.35 [20] and $0.15 \mathrm{nM}$ [21] affinity, respectively. They probably benefited from covalent immobilization instead of random binding of the target during selection to facilitate the convergence of sequences. The anti-Neu5Ac aptamer is about 60 times a stronger binder than the corresponding lectin; it also binds the derivative but about 70 times more weakly and it inhibits sialidase activity.

Different variants of SELEX, summarized in Figure 3, have been recently explored to specifically drive the in vitro selection on the glycosylation site of the biomarkers. One possibility is the incorporation of a boronic acid-modified nucleotide (boronic acid-thymidine5'-triphosphate, B-TTP) into the DNA library to boost the affinity of the aptamers [22]. This led the selection toward the glycan part of fibrinogen ( $\mathrm{K}_{\mathrm{d}}$ in the low $\mathrm{nM}$ range) in contrast to the peptide-binding aptamer resulting from conventional SELEX $\left(\mathrm{K}_{\mathrm{d}}=5 \mu \mathrm{M}\right)$. Interestingly, random introduction of B-TTP into the aptamer selected without the boronic acid moiety does not improve the binding affinity. Although no consensus in the identified sequences was found, this is a general approach to target the selection to the glycan substructure of glycoproteins.

Epitope-specific SELEX is another possibility to effectively select an aptamer against a region of a molecule. It is a special type of hybrid-SELEX, where first rounds are conventional performed and final rounds require a specific ligand competitor for elution. As an example, aptamers for the sialic acid receptor of hemagglutinin were evolved using a sialic acidcontaining protein (fetuin), so in the last rounds of selection only ssDNA bound to the sialic acid epitope in the receptor were eluted [23].

Synthesis of a short peptide containing the glycan can also serve as a target. One aptamer derived from such an approach targeted a recombinant five tandem repeats of 20 
amino acid long peptide from mucin-1 that was synthetically O-glycosylated by $15 \mathrm{~N}$ acetylgalactosamine (Tn antigen), showing affinity for both regions, peptide and sugar regions, being the affinity for Tn more than one order of magnitude higher [24]. The reason for the preference for the Tn antigen is not explained but one can speculate that their abundance (15 sugars) is the driving force. The contrary is held for aptamers derived from unglycosylated tandem peptide. This means that both types of aptamers bind a region near the glycosylated site, which is affected by the presence of the sugar. The use of synthetic peptides as targets allows the incorporation of counter-selection steps using a non-glycosylated synthetic variant, which is another possibility to direct aptamers towards the glycosylation site. This approach has been employed for the selection of a DNA aptamer that selectively bind an N-glycosylated peptide fragment of vascular endothelial growth factor (VEGF) [25]. Despite only one unit of Nacetylglucosamine is incorporated at the natural glycosylation site of VEGF, the resulting aptamers show 52-fold selectivity towards the glycan-peptide over the non-glycosylated variant. In addition, the best aptamer is able to distinguish between peptide variants differing only by a single glycosylation site. However, this study does not show the feasibility of the aptamer to recognize native VEGF, which may adopt a three-dimensional conformation different form the peptide fragment.

\section{Future perspectives}

Deciphering the glycan signature of cancer is essential to early detection and to establish personalized therapies that increase the survival rate. The challenge is extremely demanding even in the post-genomic post-proteomic era. It implies characterization of hundreds of glycans and their abundancy as well as identification of glycosylation sites. Today, many of the exact structures attached to proteins found in serum are still unknown. With this purpose, receptors recognizing the glycosylation site and capable of differentiating minimal structural features are compulsory. As it was commented above, none of the existing receptors can provide the selectivity required. Rapid advancement in glycomics reveals new aberrant 
1

2

3

4

5

6

7

8

9

alterations, which needs novel receptors. An example of this is the case of haptoglobin fucosylation associated to pathological conditions since several years. Recently, two fucosylation sites, in the core and in the sialyl determinant have been found [26]. In order to elucidate the significance of each one in any disease, specific detection is crucial. Many lectins recognize all types of linkages $\alpha 1-3,1-4$ and $\alpha 1-6$ fucosylation (core), and the most widely used specific for core fucosylation also binds mannose residues. Though a natural lectin truly specific for core fucose was found only five years ago [27] there is no suitable receptors for the rest of fucose types.

The development of synthetic receptors seems the most promising route to achieve the strict selectivity demanded. Since the biological importance of glycoproteins become clear, determined efforts are being performed to develop specific binders but there is substantial room for improvement. In this sense, aptamers could be a solution. Until now, aptamers selected against glycoproteins preferentially bind the peptide sequence unless a lot of identical sugars are present, so novel strategies directing it towards the glycan and its surrounded region are compulsory. Epitope-specific SELEX or availability of synthetic peptidoglycans can help in this task. Likewise, the synthesis of multiantennary glycans have progressed extraordinarily and now some of them are commercially available but unfortunately at not affordable fares. Moreover, the conjugation to the specific point in the peptide chain is a bottleneck for analytical chemists that need the highly purified peptidoglycan to develop better receptors. In achieving this, the availability of stronger and more specific binders will be increase.

To improve the probability of identifying aptamers with high affinity towards the glycosylation site of glycoproteins it is also possible to use chemically modified nucleobases [28]. This is the case of the so-called SOMAmers (Slow Off-rate Modified Aptamers), which contain chemically modified deoxyuridine residues with modified side chains that increase the diversity of nucleic acids thus facilitating the selection of ligands with high affinity towards 
proteins [29]. The use of high-throughput platforms such as SOMAscan [29], which has

extraordinarily accelerated the discovery of protein biomarkers, may also be useful in the field

of glycoproteins. It is conceivable that using different cross-linking chemistry aptamers

recognizing proteins but also the carbohydrate moiety, so binding near the glycosylation site, could be developed with features as good as those found in SOMAmers for detecting proteins.

Last but not least, fluent communication between analytical chemists and glycobiologists would speed up the development of improved receptors through helping chemists to early identify recognition sites of clinical/biological interest and promote less known synthetic receptors such as aptamers among clinicians or even pharmaceutical industry. The huge investment in antibodies since their discovery and the recent effort to humanize them to avoid the side effects have screened the potential of aptamers, which are ignored by the pharmaceutical and diagnostic-based industries. In the latest years, a blatant interest in aptamers is detected out of academia as a possibly solution to scientific challenges such as high degree of discrimination of minor changes not affordable with current antibodies or lectins. The detection of post-translational modifications, especially glycosylation as the most important one, is the next niche where aptamer must excel if they want to be widely recognized as a real alternative to antibodies and boost their commercialization.

Acknowledgments The authors acknowledge the support provided by the Spanish Ministerio de Economía y Competitividad (Project No. CTQ2015-63567-R), and the Principado de Asturias government (Project FC15-GRUPIN14-025, co-financed by FEDER funds).

\section{Compliance with ethical standards}

Conflict of interest The authors declare that they have not conflict of interest 
1

2

3

4

5

6

7

8

9

10

\section{REFERENCES}

1. Glavey SV, Huynh D, Reagan MR, Manier S, Moschetta M, Kawano Y, Roccaro AM, Ghobrial IM, Joshi L, O'Dwyer ME. The cancer glycome: Carbohydrates as mediators of metastasis. Blood Rev. 2015;29(4):269-79.

2. Palecek E, Tkac J, Bartosik M, Bertok T, Ostatna V, Palecek J. Electrochemistry of nonconjugated proteins and glycoproteins. Toward sensors for biomedicine and glycomics. Chem Rev. 2015;115(5):2045-108.

3. Miyoshi E, Moriwaki K, Terao N, Tan CC, Terao M, Nakagawa T, Matsumoto H, Shinzaki S, Kamada Y. Fucosylation is a promising target for cancer diagnosis and therapy. Biomolecules. 2012;2(1):34-45.

4. Vajaria BN, Patel PS. Glycosylation: a hallmark of cancer? Glycoconj J. 2017;34(2):147-56.

5. Munkley J, Elliott DJ. Hallmarks of glycosylation in cancer. Oncotarget. 2016;7(23):35478-89.

6. Shajahan A, Heiss C, Ishihara M, Azadi P. Glycomic and glycoproteomic analysis of glycoproteins- a tutorial. Anal Bioanal Chem. 2017;409(19):4483-505.

7. Jin S, Cheng YF, Reid S, Li MY, Wang BH. Carbohydrate recognition by boronolectins, small molecules, and lectins. Med Res Rev. 2010;30(2):171-257.

8. Stowell SR, Ju T, Cummings RD. Protein glycosylation in cancer. Annual Rev Pathol. 2015;10:473-510.

9. Hong X, Ma MZ, Gildersleeve JC, Chowdhury S, Barchi JJ, Jr., Mariuzza RA, Murphy MB, Mao L, Pancer Z. Sugar-binding proteins from fish: selection of high affinity "Lambodies" that recognize biomedically relevant glycans. ACS Chem Biol. 2013;8(1):152-60.

10. Tan ZJ, Yin HD, Nie S, Lin ZX, Zhu JH, Ruffin MT, Anderson MA, Simone DM, Lubman DM. Large-scale identification of core-fucosylated glycopeptide sites in pancreatic cancer serum using Mass Spectrometry. J Proteome Res. 2015;14(4):1968-78.

11. Zhou J, Yang W, Hu Y, Hoti N, Liu Y, Shah P, Sun S, Clark D, Thomas S, Zhang H. Site-specific fucosylation analysis identifying glycoproteins associated with aggressive prostate cancer cell 
lines using tandem affinity enrichments of intact glycopeptides followed by Mass Spectrometry. Anal Chem. 2017;89(14):7623-30.

12. Belicky S, Katrlik J, Tkac J. Glycan and lectin biosensors. Essays in biochemistry. 2016;60(1):37-47.

13. Sun W, Du L, Li M. Aptamer-based carbohydrate recognition. Curr Pharm Des. 2010;16(20):2269-78.

14. Dunn MR, Jimenez RM, Chaput JC. Analysis of aptamer discovery and technology. Nat Rev Chem. 2017;1:0076; doi: 10.1038/s41570-017-0076

15. de-los-Santos-Álvarez N, Lobo-Castañón MJ, Miranda-Ordieres AJ, Tuñón-Blanco P. Modified-RNA aptamer-based sensor for competitive impedimetric assay of neomycin B. J Am Chem Soc. 2007;129(13):3808-9.

16. Zhang J, Loo RRO, Loo JA. Structural characterization of a thrombin-aptamer complex by high resolution native top-down mass șSpectrometry. J Am Soc Mass Spectrom. 2017;-doi: 10.1007/s13361-017-1751-728(9):1815-22.

17. Liu Y, Kuan CT, Mi J, Zhang X, Clary BM, Bigner DD, Sullenger BA. Aptamers selected against the unglycosylated EGFRvIll ectodomain and delivered intracellularly reduce membrane-bound EGFRvIll and induce apoptosis. Biol Chem. 2009;390(2):137-44.

18. Ray P, Sullenger BA, White RR. Further characterization of the target of a potential aptamer biomarker for pancreatic cancer: cyclophilin B and its posttranslational modifications. Nucleic Acid Ther. 2013;23(6):435-42.

19. Jeong S, Eom T, Kim S, Lee S, Yu J. In vitro selection of the RNA aptamer against the Sialyl Lewis $X$ and its inhibition of the cell adhesion. Biochem Biophys Res Commun. 2001;281(1):237-43.

20. Cho S, Lee BR, Cho BK, Kim JH, Kim BG. In vitro selection of sialic acid specific RNA aptamer and its application to the rapid sensing of sialic acid modified sugars. Biotechnol Bioeng. 2013;110(3):905-13. 
1

2

3

4

5

6

7

8

9

21. Gong S, Ren HL, Tian RY, Lin C, Hu P, Li YS, Liu ZS, Song J, Tang F, Zhou Y, Li ZH, Zhang YY, Lu SY. A novel analytical probe binding to a potential carcinogenic factor of $\mathrm{N}$-glycolylneuraminic acid by SELEX. Biosens Bioelectron. 2013;49:547-54.

22. Li MY, Lin N, Huang Z, Du LP, Altier C, Fang H, Wang BH. Selecting aptamers for a glycoprotein through the incorporation of the boronic acid moiety. J Am Chem Soc. 2008;130(38):12636- $\underline{38+}+$

23. Lao YH, Chiang HY, Yang DK, Peck K, Chen LC. Selection of aptamers targeting the sialic acid receptor of hemagglutinin by epitope-specific SELEX. Chem Commun. 2014;50(63):8719-22.

24. Ferreira CS, Cheung MC, Missailidis S, Bisland S, Gariepy J. Phototoxic aptamers selectively enter and kill epithelial cancer cells. Nucleic Acids Res. 2009;37(3):866-76.

25. Rose CM, Hayes MJ, Stettler GR, Hickey SF, Axelrod TM, Giustini NP, Suljak SW. Capillary electrophoretic development of aptamers for a glycosylated VEGF peptide fragment. Analyst. 2010;135(11):2945-51.

26. Miyoshi E, Kamada Y. Application of glycoscience to the early detection of pancreatic cancer.Cancer Sci. 2016;107(10):1357-62.

27. Kobayashi Y, Tateno H, Dohra H, Moriwaki K, Miyoshi E, Hirabayashi J, Kawagishi H. A novel core fucose-specific lectin from the mushroom Pholiota squarrosa. J Biol Chem. 2012;287(41):33973-82.

28. Pfeiffer F, Rosenthal M, Siegl J, Ewers J, Mater G. Customised nucleic acid libraries for enhanced aptamer selection and performance. Curr Opin Biotechnol. 2017;48:11-18.

29. Gold L, Ayers D, Bertino J, Bock C, Bock A, Brody EN, Carter J, Dalby AB, Eaton BE, Fitzwater T et al. Aptamer-based multiplexed proteomic technology for biomarker discovery. PLoS One 2010;5:e15004. 


\section{CAPTION FOR THE FIGURES}

Fig. 1 Most common $O$ - and $N$-linked protein glycosylation and changes (highlighted in pink) typically associated to malignant transformations of cells. GlcNAc, Nacetylglucosamine. Fuc, fucose. Gal, galactose. Neu5Ac, 5-N-acetylneuraminic acid (sialic acid). GalNAc, N-acetylgalactosamine. Neu5GC, 5-N-glycolylneuraminic acid. Man, mannose

Fig. 2 Synthetic (A, B) and natural (C,D) receptors for glycan structures. (A) Boronic acid derivative. (B) Aptamer. (C) Lectin. (D) Antibody

Fig. 3 Different variant of SELEX procedure to drive the selection towards the glycosylation site of glycoproteins. (A) Use of boronic acid modified thymidine in the starting DNA library. (B) Elution with a specific ligand competitor in the last rounds of selection. (C) Counter-selection with a non-glycosylated target

Graphical Abstract: Aptamers may specifically differentiate normal from aberrant glycoproteins. 


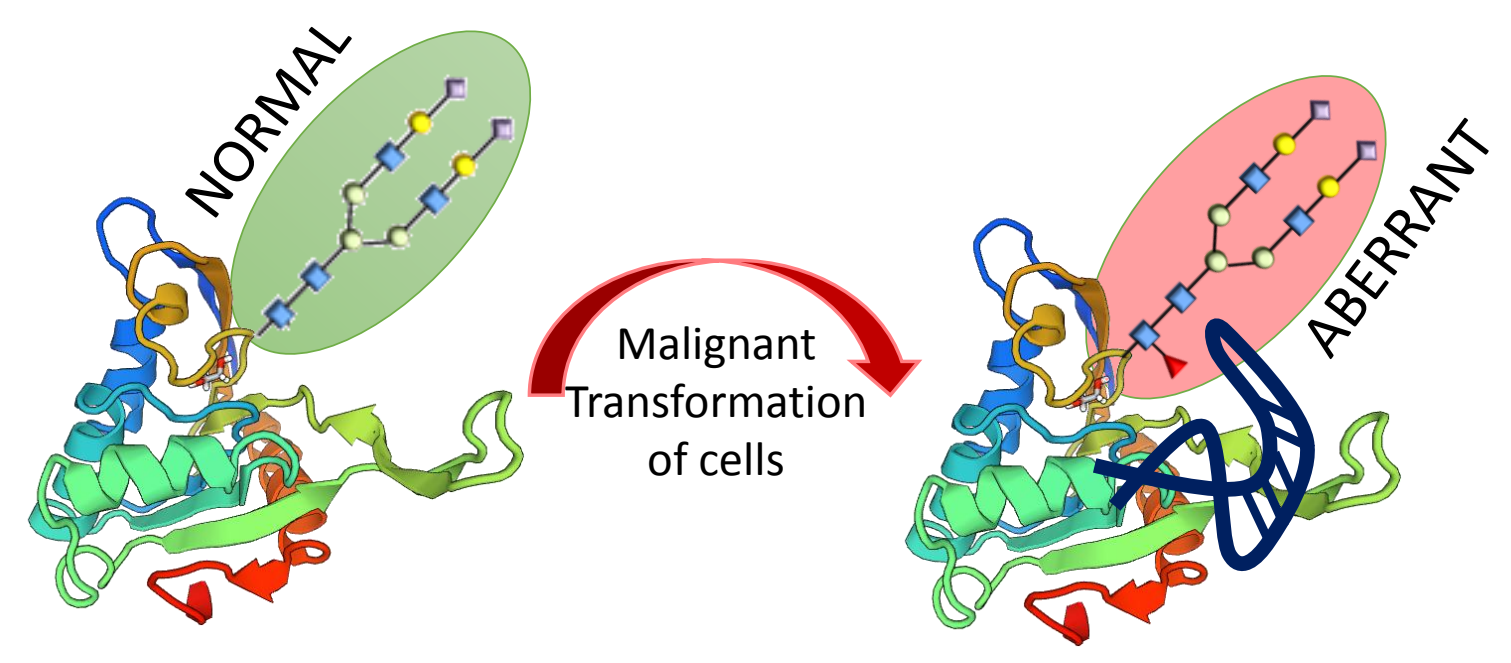



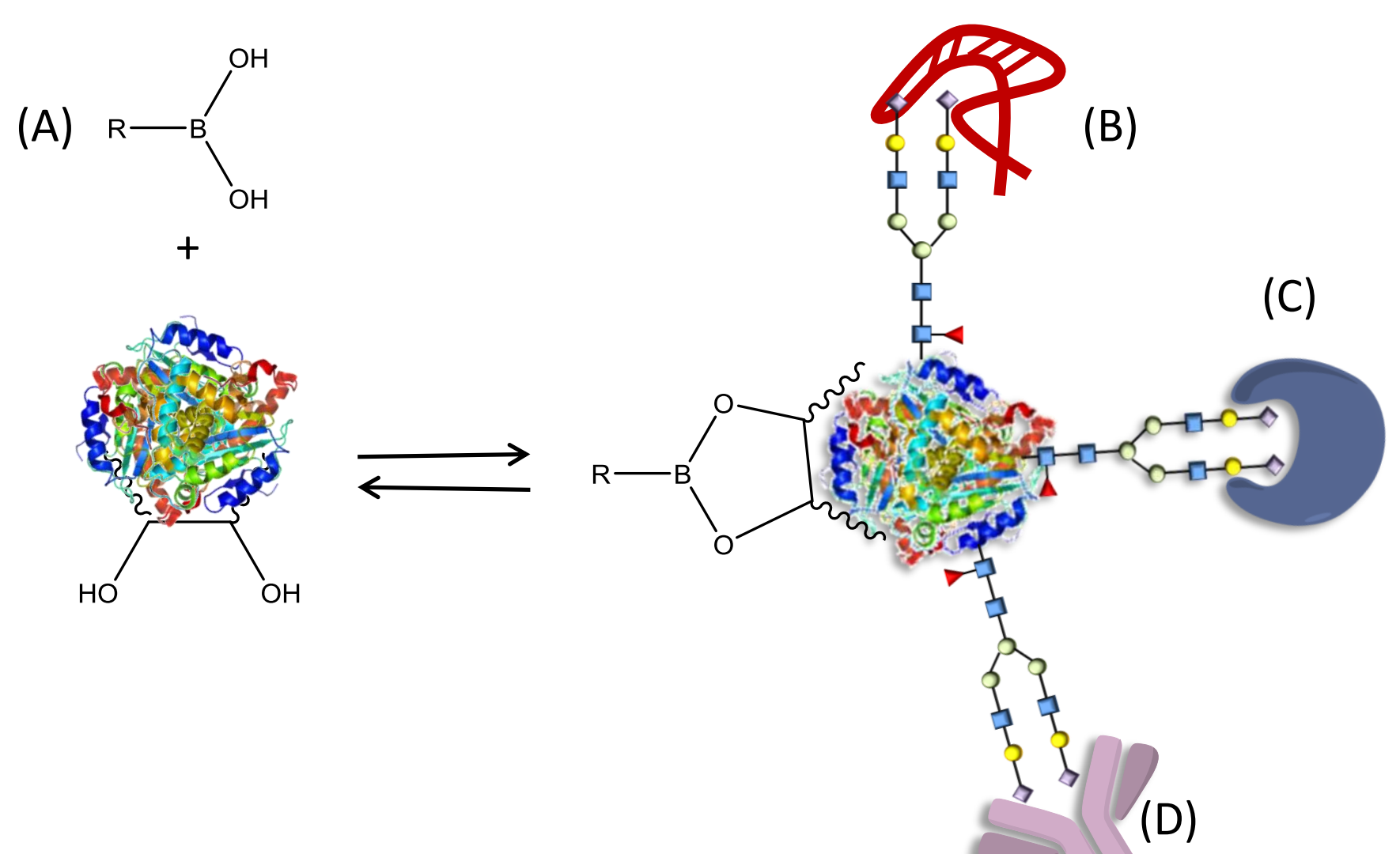


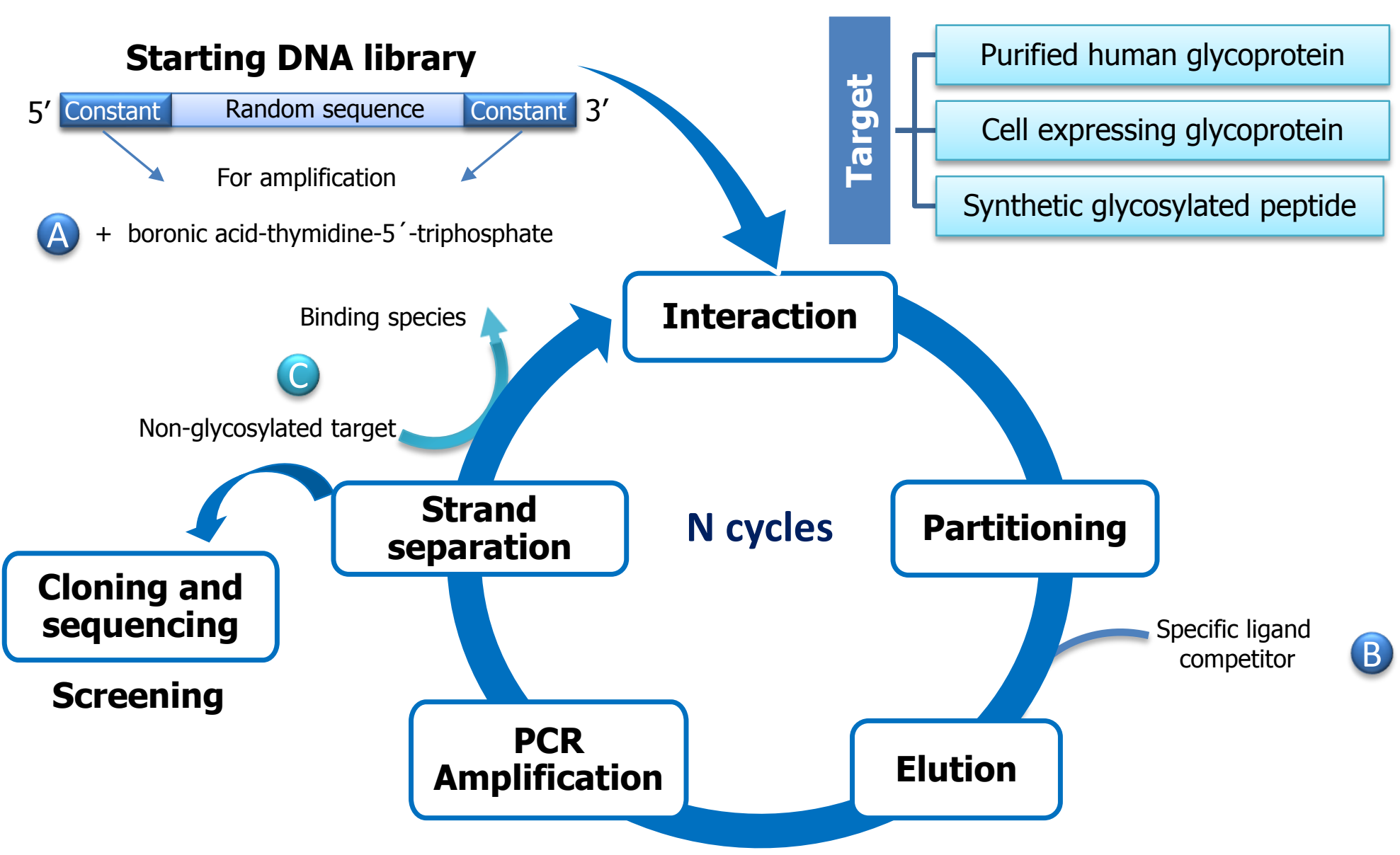

